\title{
Dislipidemias no Diabetes Melito Tipo 1: Abordagem Atual
}

\section{atuallização}

\author{
Alessandra S. de Mattos Matheus \\ Roberta Arnoldi Cobas \\ MARÍla B. GoMes
}

Disciplina de Diabetes do Departamento de Medicina Interna da Faculdade de Ciências Médicas; Hospital Universitário Pedro Ernesto (Hupe) da Universidade do Estado do Rio de Janeiro (UERJ), RJ, Brasil.

Recebido em 20/12/2007 Aceito em 10/01/2008

\section{RESUMO}

Com a intensificação do controle glicêmico no tratamento do diabetes melito tipo 1 (DM1), houve uma mudança progressiva das causas de mortalidade com destaque para a DCV. A identificação de fatores de risco, como a dislipidemia, tornou-se de grande importância para minimizar o risco de complicações crônicas micro e macrovasculares. As diretrizes para prevenção de doença coronariana em diabetes, geralmente, fazem referência ao diabetes melito tipo 2 (DM2), com pouca recomendação específica para o DM1. Definir alvos terapêuticos ou indicação de intervenção farmacológica é mais controverso nesse tipo de diabetes, em virtude da faixa etária desses pacientes. O presente estudo busca destacar a importância de estabelecer o diagnóstico da dislipidemia nesse grupo de pacientes e instituir terapêutica adequada e precoce, objetivando alcançar as metas estabelecidas para reduzir o perfil lipídico aterogênico desses pacientes. (Arq Bras Endocrinol Metab 2008;52/2:334-339)

Descritores: Diabetes melito tipo 1; Dislipidemia; Terapêutica

\section{ABSTRACT}

\section{Dyslipidemias in Type 1 Diabetes: A Current Approach.}

With the intensive glycemic control in the therapy of type 1 diabetes mellitus (T1DM) patients, cardiovascular disease has been the main cause of mortality. Identification of risk factors, such as dyslipidemia is considered of great importance in terms of avoiding chronic micro and macro vascular complications. The statements for prevention of coronary artery disease in diabetes are generally are related do type 2 diabetes mellitus and little attention is paid to T1DM. Defining therapeutical targets and indications for treatment are more controversial in these patients due to their young ages. The present study aims to emphasize the importance of establishing the diagnosis of dyslipidemia in this group of patients as well as indicate the appropriate and early treatment, in order to reach the targets of treatment and reduce the atherogenic lipid profile. (Arq Bras Endocrinol Metab 2008;52/2:334-339)

Keywords: Type 1 diabetes; Dyslipidemia; Therapeutic

\section{INTRODUÇÃO}

DIABETES MELITO TIPO l (DMl) é uma doença crônica atualmente considerada a endocrinopatia mais comum na infância e na adolescência (1). Com a intensificação do controle glicêmico e maior sobrevida desses pacientes, houve mudança progressiva das causas de mortalidade, com maior destaque para a doença cardiovascular (DCV) (2). 
Por causa da elevada morbimortalidade associada ao $\mathrm{DMl}$, com risco de complicações crônicas micro e macrovasculares precoces, torna-se importante identificar a presença de fatores de risco para DCV nessa população.

A dislipidemia na infância raramente resulta eventos adversos nessa faixa etária, porém seus efeitos na idade adulta devem ser considerados. O principal fator de risco para desenvolvimento de dislipidemia na infância, avaliados em uma metanálise de 33 estudos, foi sobrepeso ou composição corporal de gordura, sendo o sobrepeso infantil (avaliado pelo índice de massa corporal - IMC) preditor independente do desenvolvimento de dislipidemia na idade adulta (3).

A maior prevalência de sobrepeso e/ou obesidade em crianças e adolescentes vem resultando em aumento gradativo da prevalência de hipertensão arterial, dislipidemia, diabetes melito tipo 2 (DM2) em jovens $(4,5)$, assim como de DMl, sendo sugerido que, neste caso, atuasse como fator acelerador para o desenvolvimento da doença (6).

Pacientes com DMl têm risco duas a quatro vezes maior de desenvolver aterosclerose em relação à população não-diabética (2) e, nestes pacientes, os eventos cardiovasculares são responsáveis por até $44 \%$ da mortalidade total (7). Recente estudo realizado no Reino Unido observou que esses pacientes apresentaram, em todas as faixas etárias, maior mortalidade por doença arterial coronariana (8) e cerebrovascular (9) em relação à população geral. Em um estudo observacional com pacientes com DMl acompanhados por até 40 anos, $25 \%$ deles entre 30 e 55 anos apresentaram como causa de mortalidade a doença arterial coronariana (DAC), em comparação com $6 \%$ da população nãodiabética (10). O risco de mortalidade cardiovascular ajustado para idade pode até mesmo exceder o observado em pacientes com DM2 (11).

Sabe-se que crianças e adolescentes com DMl apresentam desenvolvimento precoce de doença aterosclerótica (12) com maior rigidez e espessamento de paredes arteriais comparadas aos indivíduos não-diabéticos, antes mesmo da detecção clínica de complicações micro ou macrovasculares $(13,14)$. Entretanto, nesses pacientes, $o$ ganho de peso decorrente do tratamento insulínico intensivo (15) associado às mudanças no padrão alimentar e ao sedentarismo tem sido associado à presença de um perfil lipídico mais aterogênico, com elevação de triglicerídios, LDL, ApoB e diminuição de HDL (15-17). Recentes estudos em nosso meio observaram que a prevalência de obesidade, sobrepeso e/ou risco de so- brepeso em pacientes DMl já atinge 21,2\% (18) e de síndrome metabólica varia entre $12 \%$ e $40 \%$ (19). Um subestudo de Pittsburg Epidemiology of Diabetes Complication, analisando a resistência insulínica pelo clampe euglicêmico hiperinsulinêmico, demonstrou que os principais fatores de risco para o aumento da resistência insulínica nesta população foram, em ordem decrescente de importância, relação cintural/quadril, presença de hipertensão arterial, história familiar de DM2 e níveis de HbAlc aumentados (10).

Recentemente, foi realizado um estudo de corte transversal incluindo 118 pacientes com DMl adultos acompanhados no ambulatório de diabetes da UERJ e 96 indivíduos não-diabéticos (funcionários do hospital, residentes e estudantes de medicina com glicemia de jejum $<100 \mathrm{mg} / \mathrm{dL}$ ), que teve como objetivo determinar as diferenças demográficas, clínicas ou laboratoriais entre os dois grupos. Pacientes e controles foram pareados por idade, sexo, IMC e presença de tabagismo e submetidos à avaliação clínica e laboratorial. Os pacientes com DMl não apresentaram pior perfil lipídico em relação aos controles e em geral seus níveis lipídicos são favoráveis, mesmo com controle glicêmico insatisfatório. Entretanto, estudo prévio de nosso grupo demonstrou que o subgrupo de crianças e adolescentes apresentou maior prevalência de colesterol total e LDL elevados em relação aos indivíduos não-diabéticos pareados (2).

Apesar de pacientes com DMl serem jovens e, em geral, não apresentarem alterações lipídicas importantes, as complicações micro e macrovasculares que ocorrem no DMl podem ser devidas a outros fatores que poderiam ser explicados pela hiperglicemia per se.

Dessa forma, elevações prolongadas da glicemia podem levar à glicosilação de proteínas no sangue e na parede arterial, gerando os produtos finais de glicação (AGE), o que determina aumento da concentração de espécies reativas de oxigênio e piora da função endotelial. Assim como o aumento do estresse oxidativo na célula vascular, outros processos promovidos pelo estado diabetogênico são responsáveis pela natureza prótrombótica endotelial do paciente diabético. São elas: a produção de citocinas que reduzem a produção de colágeno e tornam a placa mais vulnerável, aumento dos níveis séricos de fator de necrose tumoral-alfa e moléculas de adesão, maior produção de endotelina 1 , alteração do pool intracelular de cálcio com anormalidades plaquetárias e ativação direta de vias de sinalização intracelular pró-ateroscleróticas como os receptores para AGEs e o NFkB (20). 
Assim sendo, além da hiperglicemia, da formação acelerada de AGE avançada e do aumento do estresse oxidativo, outras condições foram propostas para explicar a aceleração da aterosclerose no diabetes, como a presença de marcadores inflamatórios (2l) e fatores genéticos (22).

Estudos em animais evidenciaram que a dislipidemia associada ao diabetes não é necessária para induzir inflamação arterial no início da lesão aterosclerótica. Em estudo experimental, o aumento do processo aterosclerótico no rato diabético pôde ser normalizado após tratamento insulínico intensivo, indicando o papel fundamental do ambiente diabetogênico, mais provavelmente associado à hiperglicemia. A hiperglicemia nos ratos com dieta isenta de colesterol induziu inflamação arterial por meio do acúmulo de macrófagos e glicosaminoglicanas na parede arterial. Estas, que se acumularam mesmo na ausência da infiltração por macrófagos, podem ser responsáveis pelo passo inicial da lesão arterial pelo diabetes (22).

Por outro lado, o aumento nos triglicerídeos ricos em VLDL induzido pelo diabetes contribui para progressão da lesão e formação de placas avançadas com hemorragia intralesional. Dessa forma, tanto a hiperglicemia quanto a dislipidemia diabética aceleram diferentes fases de aterogênese no diabetes (22).

Em relação à hiperglicemia, os estudos DCCT/ EDIC, após seguimento de 17 anos, mostraram que o tratamento intensivo do diabetes reduziu o risco de qualquer DCV em $42 \%$ e de infarto do miocárdio nãofatal, acidente vascular cerebral e morte em $57 \%$, sendo esta redução na maior parte associada à redução da hemoglobina glicada durante o DCCT (23).

Apesar de geralmente não apresentarem alterações quantitativas importantes, DMl pode apresentar alterações qualitativas na composição de suas lipoproteínas.

A participação do LDL-oxidado nesse processo tem sido apontada como fator fundamental no processo aterosclerótico em modelos animais, assim como em humanos (3). A hiperglicemia crônica resulta maior oxidação e glicação de partículas de LDL, com acúmulo de partículas densas mais aterogênicas (24).

Em estudo prévio de nosso grupo, observamos maior suscetibilidade do LDL colesterol à oxidação in vitro nos pacientes com $\mathrm{DMl}$ em relação a indivíduos não-diabéticos, sendo a hiperglicemia a única variável independente associada a este maior efeito oxidativo. Foi demonstrada que mesmo discreta glicação do LDL afeta o tônus microvascular no musculoesquelético por reduzir o diâmetro, tanto de pequenas artérias quanto de arteríolas terminais. Fato este que pode ocorrer também no estado pós-prandial (24).

Essa oxidação do LDL assume grande importância no início e na perpetuação da aterosclerose por meio da geração de lipídios inflamatórios e da modificação covalente dessa partícula. Alguns dos efeitos pró-aterogênicos do LDL modificado são a toxicidade às células endoteliais, os monócitos, o recrutamento de neutrófilos e eosinófilos e a inibição da mobilidade dos macrófagos e a promoção da formação de células espumosas (24).

Em paciente com DMl, jovens com perfil lipídico normal, o tamanho da partícula de LDL e o conteúdo de vitamina E, seu principal antioxidante, foram menores em relação a controles e associados a menor vasodilatação endotélio dependente em vasos de condutância e resistência (25).

Outros fatores parecem estar associados à doença micro e macrovascular, dos quais um dos mais importantes seria a proteína C reativa (PCR). Diferentes estudos observaram a PCR aumentada em DMl jovens e com curta duração de doença em relação a controles, sendo este aumento relacionado diretamente com os níveis de triglicerídios e razão triglicerídio/HDL. Este fato foi observado mesmo em diabéticos sem complicações micro ou macrovasculares, clinicamente detectadas, assim como o aumento da glicoproteína alfa 1 ácida (21). Sugere-se que o perfil lipídico qualitativo desfavorável no DMl favoreceria a formação de células espumosas na parede arterial com início de reação inflamatória local (26).

Conforme exposto anteriormente, é possível que altos níveis de proteínas de fase aguda em pacientes com DMl também sejam considerados um dos múltiplos fatores envolvidos na cascata de eventos da disfunção endotelial presente nesses pacientes (21).

O impacto da dislipidemia no DMl ocorre tanto na DCV quanto nas complicações microvasculares, como retinopatia e nefropatia. A retinopatia diabética $(\mathrm{RD})$ é a complicação crônica mais comum no DMl, afetando $70 \%$ a $100 \%$ dos pacientes. É conhecido o papel da hiperglicemia em seu desenvolvimento, entretanto sabe-se que o controle glicêmico intensivo não abole completamente o risco de desenvolver RD. Níveis elevados de colesterol total foram associados ao desenvolvimento de $\mathrm{RD}$ proliferativa, perda visual grave em um estudo multicêntrico realizado pela Organização Mundial de Saúde. 
Entretanto, a associação da hipertrigliceridemia com maior risco de evoluir para complicações microvasculares ainda é controverso $(27,28)$.

O papel de parâmetros do perfil lipídico na evolução para nefropatia diabética também tem sido investigado (29). Assim como na RD, o controle glicêmico não pode ser considerado único fator determinante de seu desenvolvimento. É conhecida associação entre DCV e nefropatia diabética (30), sendo a dislipidemia considerada fator de risco para desenvolvimento e progressão de ambas as condições, em virtude de a hipercolesterolemia em pacientes com DMl portadores de nefropatia diabética ser um importante preditor de evolução para insuficiência renal terminal e óbito (31).

Nos estudos Eurodiab e Diabetes Control and Complications Trial (DCCT), os valores de colesterol total e LDL foram progressivamente mais elevados com a evolução dos estágios da nefropatia diabética. Em $\mathrm{DMl}$ proteinúricos, o valor do colesterol total foi correlacionado positivamente com a velocidade de redução da taxa de filtração glomerular (32).

Recentemente, foi demonstrado que níveis mais elevados de HDL podem ser protetores para desenvolvimento de albuminúria em DMl com mais de 20 anos de duração de doença (33).

Dessa forma, o DMl está inserido em um contexto de complexos fatores que favorecem o desenvolvimento de complicações micro e macrovasculares, sendo a aterosclerose um fator de risco importante para essas complicações. No entanto, apenas o rígido controle glicêmico não parece ser suficiente para reduzir o impacto, principalmente sobre a DCV (34), devendo-se obter controle da dislipidemia com terapia adequada.

\section{BASES PARA O TRATAMENTO}

As diretrizes para prevenção de doença coronariana em diabetes, geralmente, fazem referência ao DM2 e fornecem pouca informação ou recomendação específica para o DMl. Isso, provavelmente, reflete a falta de dados disponíveis com evidências importantes. Definir alvos terapêuticos ou indicação de intervenção farmacologia é mais controverso neste tipo de diabetes em razão da faixa etária dos pacientes (34). Estudos clínicos que evidenciaram redução de risco cardiovascular em diabéticos com uso de drogas, especialmente estatinas, incluem na maior parte ou exclusivamente DM2.
Com base em dados do DCCT/EDIC, a terapia antilipêmica deve ser considerada independente do controle glicêmico, já que as taxas de eventos absolutos para cada complicação são de $2 \%$ a $5 \%$ ao ano, preenchendo critérios de risco europeus e americanos que justificam a intervenção farmacológica (34).

Um programa de curta duração ( 8 dias) de dieta apropriada recomendada pela Associação Americana de Diabetes (ADA) e atividade física ( 50 minutos, 3 vezes ao dia de exercícios aeróbicos) em DMl com controle glicêmico estável foi capaz de reduzir significativamente os níveis de colesterol total, LDL e triglicerídeos e aumentar HDL (35).

\section{AVALIAÇÃO E RASTREAMENTO}

\section{Adultos}

- Em adultos diabéticos, a avaliação deve ser realizada ao diagnóstico e então, anualmente (36), por meio da dosagem de colesterol total (CT), HDL-c, triglicerídeos (TG) após jejum de 12 a 14 horas;

- o LDL-c pode ser calculado pela equação de Friedewald $(\mathrm{LDL}=\mathrm{CT}-\mathrm{HDL}-\mathrm{TG} / 5)$ e se $\mathrm{TG}>$ $400 \mathrm{mg} / \mathrm{dl}$, obter por dosagem direta (37);

- manter dieta habitual e peso estável por pelo menos 2 semanas antes da realização do exame;

- evitar ingestão de álcool e atividade física vigorosa até 3 dias antes (37).

\section{Crianças}

- Todas as crianças maiores de 12 anos de idade ao diagnóstico. Se dentro das metas, repetir a cada 5 $\operatorname{anos}(12)$;

- todos, inclusive pré-púberes, com história familiar de DAC precoce (antes de 55 anos), DM2 ou hipertensão arterial sistêmica (HAS). Em caso de história familiar de DAC precoce ou hipercolesterolemia, o screening deve começar aos 2 anos de idade (12);

- crianças e adolescentes com níveis lipídicos anormais devem ser avaliados anualmente (36).

\section{ALVOS DO TRATAMENTO}

Em crianças, os níveis de LDL são a base para iniciar o tratamento e determinar os alvos terapêuticos, os quais devem ser mantidos $<100 \mathrm{mg} / \mathrm{dl} \mathrm{(3)}$. 
Em adultos, os alvos terapêuticos são melhores definidos, sendo primeiramente os níveis de LDL $(<100$ $\mathrm{mg} / \mathrm{dl}$ ), e, secundariamente, de HDL ( $>50 \mathrm{mg} / \mathrm{dl}$ ) e triglicerídeos $(<150 \mathrm{mg} / \mathrm{dl})(8)$. Sugere-se níveis de $\mathrm{LDL}<70 \mathrm{mg} / \mathrm{dl}$ para portadores de doença aterosclerótica clínica $(36)$.

Obtenção de níveis de LDL-c abaixo de $100 \mathrm{mg} / \mathrm{dl}$ (2,6 mmol/l) é mais consistente para prevenção de DAC, sendo níveis abaixo de $130 \mathrm{mg} / \mathrm{dl}(3,3 \mathrm{mmol} / \mathrm{L})$ aceitáveis para outras complicações. Níveis de triglicerídeos abaixo de $150 \mathrm{mg} / \mathrm{dl}(2,3 \mathrm{mmol} / \mathrm{L})$ são consistentes em diminuir o risco de mortalidade, DAC e nefropatia diabética. Com exceção da DAC, níveis de HDL-C $\geq 45 \mathrm{mg} /$ $\mathrm{dl}(1,1 \mathrm{mmol} / \mathrm{L})$ não apresentaram forte evidência de prevenção de outras complicações crônicas (34).

\section{INDICAÇÕES DO TRATAMENTO}

Todos os diabéticos, independente da idade, com hipercolesterolemia familiar e hiperlipidemia combinada familiar devem ser tratados (38), assim como indivíduos maiores de 40 anos, com outros fatores de risco (36).

\section{Tratamento específico}

Modificações no estilo de vida, com aumento de atividade física, dieta adequada com redução da ingestão de calorias, gorduras saturadas e colesterol, e terapia farmacológica (36).

Os resultados das intervenções não farmacológicas devem ser reavaliados a cada 3 ou 6 meses, para então determinar a necessidade de instituir terapia medicamentosa, quando os alvos não forem atingidos. Entretanto, em pacientes com DCV estabelecida e LDL > $100 \mathrm{mg} / \mathrm{dl}$ ou aqueles sem DCV mas com LDL > 130 $\mathrm{mg} / \mathrm{dl}$, a terapia medicamentosa deve ser iniciada juntamente às medidas não-farmacológicas (36).

Para crianças acima de 2 anos de idade é aprovado a instituição de dieta com baixo teor de gorduras e colesterol, como a dieta step I da American Heart Association (3). Entretanto para crianças menores de 2 anos não devem ser prescritas dietas com baixo teor de gordura e colesterol em razão da necessidade nutricional nesta faixa etária de crescimento e desenvolvimento rápidos.

Para crianças com menos de 8 anos resina quelante de ácidos biliares (colestiramina) é a única droga aprovada (12). Porém sua baixa palatabilidade e sintomas, como constipação, flatulência, dor abdominal, tornam sua tolerabilidade baixa (3).
Entre os inibidores da HMG-CoA redutase, a atorvastatina é a única aprovada para uso em crianças com mais de 10 anos com hipercolesterolemia familiar heterozigótica.

Para pacientes adultos que não atingem níveis ótimos apesar da terapia com estatinas, deve-se considerar a associação de drogas: estatina com ezetimibe ou com fibrato ou com niacina (38). Neste caso deve-se estabelecer vigilância quanto a efeitos adversos e toxicidade.

\section{Contra-indicações}

Em crianças é contra-indicado o uso de niacina ou fibratos (38).

Atenção especial deve ser dada a mulheres em idade reprodutiva.

\section{CONSIDERAÇÕES FINAIS}

Pacientes com DMl representam uma população de alto risco para desenvolvimento de doenças cardiovasculares e mortalidade precoce. Entretanto, por serem jovens, muitas vezes o tratamento da dislipidemia é postergado na prática clínica. O presente estudo busca destacar a importância de estabelecer o diagnóstico da dislipidemia neste grupo de pacientes e instituir terapêutica adequada, atingindo metas recomendadas no intuito de minimizar os efeitos deletérios de um perfil lipídico aterogênico.

\section{REFERÊNCIAS}

1. American Diabetes Association. Diabetes statistics for Youth. Disponível em: http://www.diabetes.org/utils/printthispage. jsp, 2004.

2. Arcanjo CL, Piccirillo LJ, Machado IV, Andrade CRM, Clemente $\mathrm{EL}$, Gomes MB. Avaliação de dislipidemia e de índices antropométricos em pacientes com diabetes mellitus tipo 1. Arq Bras Endocrinol Metab. 2005;49(6):951-8.

3. Haney EM, Huffman LH, Bougatsos C, Freeman M, Steiner RD, Nelson HD. Screening and treatment for lipid disorders in children and adolescents: systematic evidence review for the US Preventive Services Task Force. Pediatrics. 2007;120(1): e189-207.

4. Berenson GS, Srinivasan SR, Bao W, Newman III WP, Tracy RE, Wattigney WA. Association between multiple cardiovascular risk factors and atherosclerosis in children and young adults. N Eng J Med. 1998;338:1650-6.

5. Invitti C, Guzzaloni G, Gilardini L, Morabito F, Viberti G. Prevalence and comcomitants of glucose intolerance in European obese children and adolescents. Diabetes Care. 2003;26:118-24.

6. Libman IM, Pietropaolo M, Arslanian SA, LaPorte RE, Becker DJ. Changing Prevalence of overweight chilidrem and adolescents at onset of Insulin-treated Diabetes. Diabetes Care. 2003;26:2871-5. 
7. Rassi N, Rassi TO. Síndrome metabólica no diabetes tipo 1. In: Ruy Lira, Ney Cavalcanti, organizadores. Diabetes mellitus. $1^{\underline{a}}$ ed. BR: Diagraphic; 2006. p. 208.

8. Laing SP, Swerdlow AJ, Slater SD, Burden AC, Morris A, Waugh NR, et al. Mortality from heart disease in a cohort of 23,000 patients with insulin-treated diabetes. Diabetologia. 2003;46:760-5.

9. Laing SP, Swerdlow AJ, Carpenter LM, Slater SD, Burden AC, Botha JL, et al. Mortality from cerebrovascular disease in a cohort of 23000 patients with insulin-treated diabetes. Stroke. 2003;34:418-21.

10. Malcom GT, Oalmann MC, Strong JP. Risk factors for atherosclerosis in young subjects: the PDAY study (Pathological Determinants of Atherosclerosis in Youth). Ann NY Acad Sci. 1997;817:179-88.

11. Libby P, Nathan DM, Abraham K, Brunzell JD, Fradkin JE, Haffner SM, et al. Report of the national heart, lung, and blood Institute-National Institute of Diabetes and Digestive and Kidney Diseases Working Group on Cardiovascular Complications of Type 1 Diabetes Mellitus. Circulation. 2005;111:3489-93.

12. Dahl-Jorgensen K, Larsen JR, Hanssen KF. Atherosclerosis in childhood and adolescent type 1 diabetes: early disease, early treatment? Diabetologia. 2005;48:1445-53.

13. Rönnback M, Fagerudd J, Forsblom C, Pettersson-Fernholm K, Reunanen A. Per-Henrik Groop and on behalf of the finnish diabetic nephropathy. Altered age-related blood pressure pattern in type 1 diabetes. Circulation. 2004;110:1076-82.

14. Giannattasio C, Failla M, Piperno A, Grappiolo A, Gamba P, Paleari $F$, et al. Early impairment of large artery structure and function in Type I diabetes mellitus. Diabetologia. 1999;42:987-94.

15. Purnell JQ, Hokanson JE, Marcovina SM, Steffes MW, Cleary PA, Brunzell JD. Effect of excessive weight-gain with intensive therapy of type 1 diabetes on lipid levels and blood pressure: results from DCCT. Diabetes control and complications trial. JAMA. 1998;280:140-6.

16. Lemos MCC. Dieta e dislipidemias. In: Francisco Bandeira, Endocrinologia e diabetes. $1^{\text {a }}$ ed. Rio de Janeiro: MEDSI; 2003; p. 1067.

17. Rader DJ, Davidson MH, Caplan RJ, Pears JS. Lipid and apolipoprotein ratios: associaton with coronary artery disease and effects of rosuvastatin compared with atrovastatin, pravastatin, and simvastatin. Am J Cardiol. 2003;91(5A):20C-4C.

18. Moraes CM, Portella RB, Pinheiro VS, et al. Prevalência de sobrepeso e obesidade em pacientes com diabetes tipo 1. Arq Bras Endocrinol Metab. 2003;47(6):677-83.

19. Dib SA. Resistência à insulina e síndrome metabólica no diabetes melito tipo 1. Arq Bras Endocrinol Metab. 2006;50(2):250-63.

20. Rassi N, Rassi TO. Dislipidemia diabética da etiologia ao tratamento In: Ruy Lira, Ney Cavalcanti, organizadores. Diabetes mellitus. 1aㅡ. ed. Rio de Janeiro: Diagraphic; 2006; p. 587.

21. Gomes MB, Piccirillo LJ, Nogueira VG, Matos HJ. Acute-phase proteins among patients with type 1 diabetes. Diabetes Metab. 2003;29:405-11.

22. Renard CB, Kramer F, Johansson F, Lamharz N, Tannock LR, et al. Diabetes and diabetes-associated lipid abnormalities have distinct effects on initiation and progression of atherosclerotic lesions. J Clin Invest. 2004;114(5):659-68.

23. The Diabetes Control and Complications Trial/Epidemiology of Diabetes Interventions and Complications (DCCT/EDIC) Study Research Group. Intensive diabetes Treatment and cardiovascular Disease in patients with type 1 diabetes. N Engl J Med. 353;25:2643-53.
24. Castro SH, Faria-Neto HCC, Gomes MB. Association of postprandial hyperglycemia with in vitro LDL oxidation in nonsmoking patients with type 1 diabetes - a cross-sectional study. Rev Diabetic Stud. 2005;2(3):157-64.

25. Skyrme-Jones RA, O'Brien RC, Luo M, Meredith IT. Endothelial Vasodiltators function is related to low-density lipoprotein particle size and low-density lipoprotein vitamin E content in type 1 diabetes. J Am Coll Cardiol. 2000;35:292-9.

26. Ladeia Am, Stefanelli E, Ladeia-Frota C, Moreira A, Hiltner A, Adan L. Association between elevated serum C-reactive protein and triglyceride levels in young subjects with type 1 diabetes. Diabetes Care. 2006;29:424-5.

27. Boelier MC, Azevedo MJ, Gross JL, Lavinski J. Fatores de risco para retinopatia diabética. Arq Bras Oftalm. 2003;66:239-47.

28. Chaturvedi N, Sjoelie AK, Aldington SJ, Fuller JH, Songini M, Kohner EM. Markers of insulin resistence are strong risk factors for retinopathy incidence in type 1 diabetes. The EURODIAB porspective complications study. Diabetes Care. 2001; 24:284-9.

29. Gomes, MB, Lucchetti MR, Gazolla H, Dimetz T, Lobão VI, Stum J A. Perfil lipídico, microalbuminúria e pressão arterial sistêmica em pacientes com diabetes insulino-dependente. Arq Bras Cardiol. 1997;68(2):85-9.

30. Tuomilehto J, Borch-Johnsen K, Molarius A, Forsem T, Rastenyte $D$, Sarti $C$, et al. Incidence of cardiovascular disease in type 1 (insulin-dependent) diabetic subjects with and without diabetic nephropathy in Finland. Diabetologia. 1998;41:784-90.

31. Krolewski AS, Warram JTT, Christlieb R. Hypercholesterolemia. A determinant of renal function loss and death in IDDM patients with nephropathy. Kidney Int. 1994;45(Suppl 45): S125-31.

32. Chatuverdi N, Fuller JH, Taskinen MR. Differing associations of lipid and lipoprotein disturbances with the macrovascular and microvascular complications ot type I diabetes Diabetes Care. 2001;24:2071-7.

33. Molitch ME, Rupp D, Carnethon M. Higher levels of HDL cholesterol are associated with a decreased likelihood of albuminuria in patients with long-standing type 1 diabetes. Diabetes Care. 2006;29:78-82.

34. Orchard TJ, Forrest KYZ, Kuller LH, Becker DJ. Lipid and blood pressure treatment goals for type 1 diabetes: 10-year incidence data from the Pittsburgh Epidemiology of Diabetes Complications Study. Diabetes Care. 2001;24(6):1053-8.

35. Khawali C, Andriolo A, Ferreira SR. Benefícios da atividade física no perfil lipídico de pacientes com diabetes tipo 1. Arq Bras Endocrinol Metab. 2003;47(1).

36. Posicionamento oficial da SBD $n \circ 5$. Dislipidemia no paciente diabético: aspectos etiopatogênicos, clínicos e terapêuticos. Rev Bras Med. Suplemento especial 5/2007.

37. IV Diretriz Brasileira sobre Dislipidemias e Prevenção da Aterosclerose. Departamento de Aterosclerose da Sociedade Brasileira de Cardiologia. Arq Bras Cardiol. 2007;88(Suppl I).

38. Furtado MV, Polanczyk CA. Prevenção cardiovascular em pacientes com diabetes: revisão baseada em evidências. Arq Bras Endoc Metab. 2007;51(2):312-8.

\section{Endereço para correspondência:}

Alessandra Saldanha de Mattos Matheus

Rua João Pessoa, 197/1201

24220-330 Icaraí Niterói, RJ

E-mail: marialia@uerj.br 